\title{
UP-DOWN COLORINGS OF VIRTUAL-LINK DIAGRAMS AND THE NECESSITY OF REIDEMEISTER MOVES OF TYPE II
}

\author{
KANAKO OSHIRO, AYAKA SHIMIZU, AND YOSHIRO YAGUCHI
}

\begin{abstract}
We introduce an up-down coloring of a virtual-link diagram. The colorabilities give a lower bound of the minimum number of Reidemeister moves of type II which are needed between two 2-component virtual-link diagrams. By using the notion of a quandle cocycle invariant, we determine the necessity of Reidemeister moves of type II for a pair of diagrams of the trivial virtual-knot. This implies that for any virtual-knot diagram $D$, there exists a diagram $D^{\prime}$ representing the same virtual-knot such that any sequence of generalized Reidemeister moves between them includes at least one Reidemeister move of type II.
\end{abstract}

\section{INTRODUCTION}

Necessity of Reidemeister moves, or estimations of the minimum number of those moves, between two diagrams of the same classical-knot or classical-link have been studied by several ways; for example, for studies using the geometrical feature of diagrams or their Gauss diagram, see [6, 7, 8, 9, 12, 13, 16, for studies using the concepts of quandle colorings and quandle cocycle invariants, see [1, 3].

In terms of Reidemeister moves of type II, by using geometric properties, T. J. Hagge [6] and V. O. Manturov [12] costructed pairs of diagrams of the same classical-knot such that at least one Reidemeister moves of type II is needed between them. Especially, Hagge proved that every classical-knot admits a pair $\left(D, D^{\prime}\right)$ of diagrams such that any sequence of Reidemeister moves between $D$ and $D^{\prime}$ includes a Reidemeister move of type II. On the other hand, as far as we know, there is no method using the concept of quandle colorings or quandle cocycle invariants to determine the necessity of Reidemeister moves of type II. 1]

In this paper, we study about the necessity (or an estimation of the minimum number) of Reidemeister moves of type II for virtual-link diagrams by using up-down colorings and the notion of quandle cocycle invariants. Note that our method is also useful for classical-link diagrams, that is, the readers may read all the part except for Theorem 1.4 by replacing "virtual" with "classical" and "generalized Reidemeister moves" with "Reidemeister moves". Besides, in this paper, a virtual-link (or virtualknot) diagram means an oriented virtual-link (or virtual-knot) diagram.

For a virtual-link (or virtual-knot) diagram $D$, we introduce the number of $n$ up-down colorings $\# \operatorname{Col}_{n}(D)$ which is analogous to quandle coloring numbers, the

The first author was supported by JSPS KAKENHI Grant Number 16K17600.

The second author was supported by Grant for Basic Science Research Projects from The Sumitomo Foundation (160154).

${ }^{1}$ Z. Cheng and H. Gao [3] also studied about the necessity of Reidemeister moves of type I I by using the concept of quandle colorings. However, their method contains a key error in the conditions of an algebraic structure. 
maximum order of up-down colorings maxord $(D)$, and a multi-set $\Phi_{f}(D)$ which is analogous to quandle cocycle invariants. We show the following theorems:

Theorem 1.1. Let $D$ and $D^{\prime}$ be diagrams which represent the same virtual-link. If $\# \operatorname{Col}_{n}(D) \neq \# \operatorname{Col}_{n}\left(D^{\prime}\right)$, then any sequence of generalized Reidemeister moves between $D$ and $D^{\prime}$ includes at least one Reidemeister move of type II.

Theorem 1.2. Let $D$ and $D^{\prime}$ be 2-component virtual-link diagrams which represent the same virtual-link. Any finite sequence of generalized Reidemeister moves between $D$ and $D^{\prime}$ includes at least $\left|\operatorname{maxord}(D)-\operatorname{maxord}\left(D^{\prime}\right)\right| / 2$ Reidemeister moves of type II.

Theorem 1.3. Let $D$ and $D^{\prime}$ be diagrams which represent the same virtual-knot. If $\Phi_{f}(D) \neq \Phi_{f}\left(D^{\prime}\right)$ as multi-sets, then any sequence of generalized Reidemeister moves between $D$ and $D^{\prime}$ includes at least one Reidemeister move of type II.

As an application of Theorem 1.3 we have the following result.

Theorem 1.4. For any virtual-knot diagram $D$, there exists a diagram $D^{\prime}$ representing the same virtual-knot such that any sequence of generalized Reidemeister moves between $D$ and $D^{\prime}$ includes at least one Reidemeister move of type II.

The rest of the paper is organized as follows: In Section 2, we review the definitions of virtual-links and the generalized Reidemeister moves. In Section 3, we introduce up-down colorings for virtual-link diagrams and prove Theorems 1.1 and 1.2. In Section 4 , we define a multi-set $\Phi_{f}(D)$ which is analogous to quandle cocycle invariants and prove Theorems 1.3 and 1.4 .

\section{VIRTUAL-LINKS}

A classical-link with $r$-components is $r$ circles embedded in $\mathbb{R}^{3}(r=1,2, \ldots)$. We call a classical-link with $r=1$ a classical-knot. A diagram of a classical-link $L$ is the image $p(L)$ of $L$ by a regular projection $p: \mathbb{R}^{3} \rightarrow \mathbb{R}^{2}$ with over/under information at each crossing. We call such a crossing a real-crossing, see the left picture of Figure 1. It is known that two classical-link diagrams represent the same
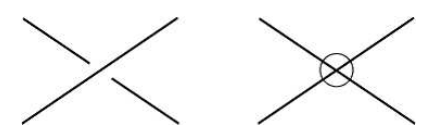

Figure 1. A real-crossing and a virtual-crossing.

classical-link if and only if there exists a finite sequence of the Reidemeister moves of type I, type II or type III between them, where the Reidemeister moves are the local transformations (two RIs, an RII and two RIIIs) on classical-link diagrams depicted in Figure 2, see 14.

A virtual-link diagram is generic closed curves in $\mathbb{R}^{2}$ each of whose double points is a real-crossing or a virtual-crossing depicted in Figure 1. We call each closed curve a component of the virtual-link diagram, and a virtual-link diagram with $r$-components an $r$-component virtual-link diagram. A 1-component virtual-link diagram is also called a virtual-knot diagram. It is said that two virtual-link diagrams represent the same virtual-link if there exists a finite sequence of the generalized 

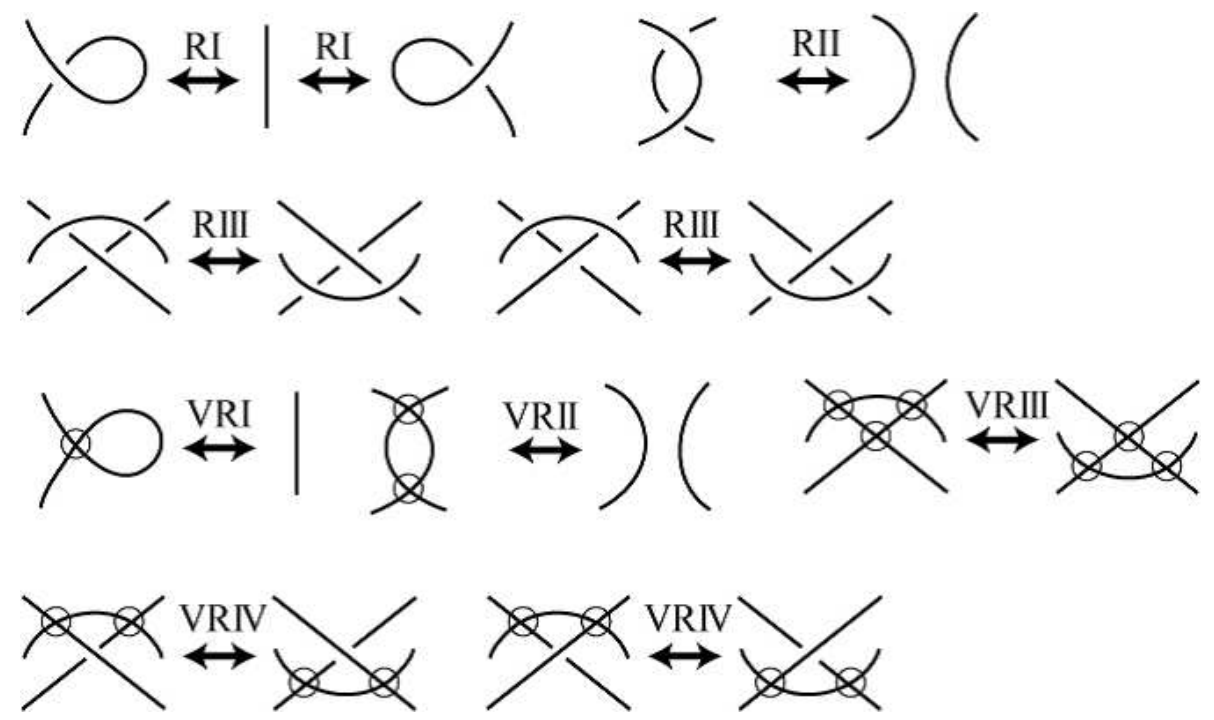

Figure 2. The Generalized Reidemeister moves.

Reidemeister moves which are depicted in Figure 2 between them. In this way, classical-links are expanded to virtual-links. For more details, see [10].

In this paper, we assume that virtual-link diagrams are oriented, and then, generalized Reidemeister moves mean oriented generalized Reidemeister moves. We call a Reidemeister move of type I, of type II and of type III an RI-move, an $R I$ $I$-move and an RIII-move, respectively. Similarly, for the other generalized Reidemeister moves, we call them a VRI-move, a VRII-move, a VRIII-move and a VRI $V$-move, respectively.

\section{UP-DOWN COLORINGS AND THE NECESSITY OF REIDEMEISTER MOVES OF TYPE II}

We note again that we may read this section by replacing "virtual" with "classical" and "generalized Reidemeister moves" with "Reidemeister moves".

In this section, we define an up-down coloring for a virtual-link diagram and investigate its properties.

Let $n$ be a positive integer and $\mathbb{Z}_{n}$ the cyclic group $\mathbb{Z} / n \mathbb{Z}$.

Let $D$ be a virtual-link diagram. The diagram $D$ is separated into the small edges such that the end points of each edge are real-crossings and there is no realcrossing in the interior of each edge. We call such an edge a semi-arc of $D$. Let $\mathcal{S} \mathcal{A}(D)$ denote the set of semi-arcs of $D$.

Definition 3.1. An $n$-up-down coloring of $D$ is a map $C: \mathcal{S A}(D) \rightarrow \mathbb{Z}_{n}$ which satisfies the following condition: For a real-crossing $c$ of $D$, let $u_{1}, u_{2}$ (resp. $o_{1}, o_{2}$ ) be the under-semi-arcs (resp. over-semi-arcs) around $c$ such that the orientation of $D$ points from $u_{1}$ to $u_{2}$ (resp. from $o_{1}$ to $o_{2}$ ). Then it holds that

$$
C\left(u_{2}\right)=C\left(u_{1}\right)-1 \text { and } C\left(o_{2}\right)=C\left(o_{1}\right)+1 .
$$

See Figure 3. When we do not specify $n$, we also call it an up-down coloring of $D$ for simplicity. For each semi-arc $e$ of $D$, we call $C(e)$ the color of $e$. 


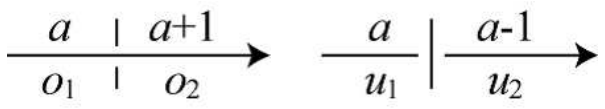

Figure 3. The conditions of an up-down coloring.

Remark. 3.2. Up-down colorings are regarded as a generalization of the warping degree labellings defined in [15], and see also [11.

We denote by $\operatorname{Col}_{n}(D)$ the set of $n$-up-down colorings of $D$. Since $\mathcal{S A}(D)$ and $\mathbb{Z}_{n}$ are finite sets, so is $\operatorname{Col}_{n}(D)$. Therefore, we call the cardinality of $\operatorname{Col}_{n}(D)$ the number of $n$-up-down colorings of $D$, and denote it by $\# \operatorname{Col}_{n}(D)$.

Now, we consider about the numbers of $n$-up-down colorings in the cases of virtual-"knots".

Lemma 3.3. For any virtual-knot diagram $D, \# \operatorname{Col}_{n}(D)=n$.

Proof. Choose a semi-arc $e$ of $D$ and fix it. We pass through the over-crossings as many as the under-crossings while we travel along the diagram $D$ from $e$ to $e$ according to the orientation of $D$. This implies that for any $a \in \mathbb{Z}_{n}$, we have a unique up-down coloring of $D$ such that the color of $e$ is $a$. Therefore the number of $n$-up-down colorings of $D$ coincides with that of choices of elements of $\mathbb{Z}_{n}$.

Moreover, we have the following lemma:

Lemma 3.4. Let $D$ be a virtual-knot (or virtual-link) diagram and $C$ an n-up-down coloring of $D$. Set a map $C^{\prime}: \mathcal{S A}(D) \rightarrow \mathbb{Z}_{n}$ by $C^{\prime}(e)=C(e)+1$ for e $\in \mathcal{S} \mathcal{A}(D)$. Then the map $C^{\prime}$ is also an n-up-down coloring of $D$.

Proof. By using the equation (11) of the condition of an up-down coloring, we have

$$
C^{\prime}\left(u_{2}\right)=C\left(u_{2}\right)+1=\left(C\left(u_{1}\right)-1\right)+1=\left(C\left(u_{1}\right)+1\right)-1=C^{\prime}\left(u_{1}\right)-1
$$

and

$$
C^{\prime}\left(o_{2}\right)=C\left(o_{2}\right)+1=\left(C\left(o_{1}\right)+1\right)+1=C^{\prime}\left(o_{1}\right)+1 .
$$

Therefore $C^{\prime}$ also satisfies the condition (1) of an up-down coloring.

By Lemmas 3.3 and 3.4 next property holds:

Corollary 3.5. For a virtual-knot diagram $D$ and an $n$-up-down coloring $C$ of $D$,

$$
\operatorname{Col}_{n}(D)=\left\{C+i \mid i \in \mathbb{Z}_{n}\right\},
$$

where $C+i$ is the map from $\mathcal{S} \mathcal{A}(D)$ to $\mathbb{Z}_{n}$ which maps a semi-arc e to $C(e)+i$.

Next, let us consider about the numbers of $n$-up-down colorings in the cases of virtual- "links". In the cases of virtual-links with at least 2-components, the number of $n$-up-down colorings depends on the choice of a diagram $D$. For example, $T(1)$ does not have a 4-up-down coloring, while $T(2)$ does, where $T(1)$ and $T(2)$ are the virtual-link diagrams, representing the same virtual-link, depicted in Figure 5 , However, Theorem 1.1] shows that the numbers of $n$-up-down colorings are useful to detect the necessity of RII-moves.

Proof of Theorem 1.1. It suffices to show that the number of $n$-up-down colorings of a virtual-link diagram is unchanged under the generalized Reidemeister moves except for the RII-moves. 
Let $D$ and $D^{\prime}$ be virtual-link diagrams such that $D^{\prime}$ is obtained from $D$ by a single generalized Reidemeister move other than the RII-moves. Let $E$ be a 2-disk in $\mathbb{R}^{2}$ in which the move is applied. Let $C$ be an $n$-up-down coloring of $D$. We define an $n$-up-down coloring $C^{\prime}$ of $D^{\prime}$, corresponding to $C$, by $C^{\prime}(e)=C(e)$ for a semi-arc $e$ appearing in $\mathbb{R}^{2}-E$. Then the colors of the semi-arcs appearing in $E$, by $C^{\prime}$, are uniquely determined, see Figure 13 for the RI-moves, Figure 14 for the RIII-moves and Figure 15 for the VRIV-moves. Thus we have a bijection

$$
\mathrm{Col}_{n}(D) \rightarrow \mathrm{Col}_{n}(D) ; C \mapsto C^{\prime} .
$$

A virtual-link diagram $D$ is $n$-up-down colorable if there exists an $n$-up-down coloring of $D$. The maximum order of up-down colorings of a virtual-link digram $D$ is the maximum number of positive integers $n$ such that $D$ is $n$-up-down colorable if it is finite, and 0 otherwise. We denote it by $\operatorname{maxord}(D)$. By Theorem 1.2, we give an estimation of the minimum number of required RII-moves between given two 2-component virtual-link diagrams.

Proof of Theorem 1.2. By the proof of Theorem 1.1, it is easy to show that the generalized Reidemeister moves other than the RII-moves do not change the maximum order of up-down colorings. Hence, it is sufficient to show that for virtual-link diagrams $D$ and $D^{\prime}$ which are related by a single RII-move, the value $\left|\operatorname{maxord}(D)-\operatorname{maxord}\left(D^{\prime}\right)\right| / 2$ is at most one.

Let $D$ and $D^{\prime}$ be virtual-link diagrams such that $D^{\prime}$ is obtained from $D$ by the RII-move shown in Figure 4 Let $E$ be a 2-disk in $\mathbb{R}^{2}$ in which the move is applied. As in Figure 4, we denote by $e_{1}$ and $e_{2}$ (or $e_{1}^{\prime}$ and $e_{2}^{\prime}$ ) two semi-arcs of $D$ (or of $D^{\prime}$ ) appeared in $E$. Here, we assume that $e_{1}$ and $e_{2}$ (or $e_{1}^{\prime}$ and $e_{2}^{\prime}$ ) are in the distinct components. By traveling along one component of the diagram $D$ from $e_{1}$ to $e_{1}$ according to the orientation of $D$, we read the colors of the semiarcs which are passed through. Thus we can see that $D$ is $n$-up-down colorable if and only if $C\left(e_{1}\right)=C\left(e_{1}\right)+o-u$ in $\mathbb{Z}_{n}$ (and $C\left(e_{2}\right)=C\left(e_{2}\right)-o+u$ in $\mathbb{Z}_{n}$ ) for some $n$-up-down coloring $C$, see Figure 4, where $o$ and $u$ is the numbers of the non-self over-crossings and the non-self under-crossings, respectively, which we passed through. This implies that $\operatorname{maxord}(D)=|o-u|$. On the other hand, the numbers of the non-self over-crossings and the non-self under-crossings which are passed through while we travel along one component of the diagram $D^{\prime}$ from $e_{1}^{\prime}$ to $e_{1}^{\prime}$ according to the orientation of $D^{\prime}$ are $o$ and $u+2$, respectively. Thus $\operatorname{maxord}\left(D^{\prime}\right)=|o-(u+2)|=|o-u-2|$ holds. Hence we have

$$
\operatorname{maxord}(D)-2=|o-u|-2 \leq|o-u-2|=\operatorname{maxord}\left(D^{\prime}\right),
$$

and

$$
\operatorname{maxord}\left(D^{\prime}\right)=|o-u-2| \leq|o-u|+2=\operatorname{maxord}(D)+2 .
$$

Therefore it holds that

$$
\left|\operatorname{maxord}(D)-\operatorname{maxord}\left(D^{\prime}\right)\right| / 2 \leq 1 .
$$

Similarly, we can see that in the case that $e_{1}$ and $e_{2}$ (or $e_{1}^{\prime}$ and $e_{2}^{\prime}$ ) are in the same component, we have

$$
\left|\operatorname{maxord}(D)-\operatorname{maxord}\left(D^{\prime}\right)\right| / 2=0 \leq 1 .
$$


For the other RII-moves, we also have the same inequality.

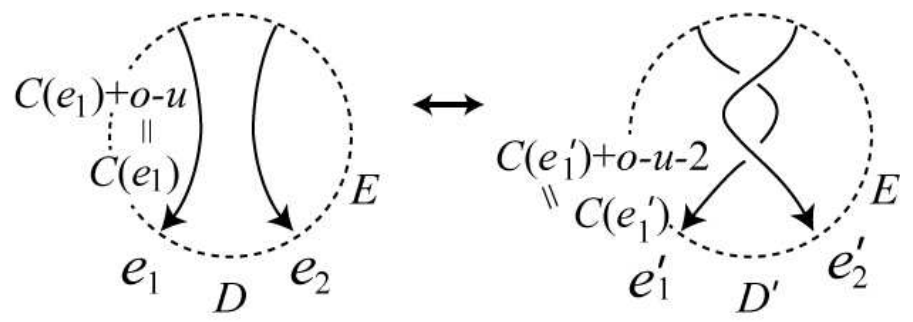

FIgURE 4. An RII-move and colorability by up-down colorings.

Example 3.6. Let $\{T(i)\}_{i \in\{0,1,2, \ldots\}}$ be the family of the virtual-link diagrams depicted in Figure 5. When $i \in\{0,1,2, \ldots\}, T(i)$ is $n$-up-down colorable if and only if $2 i \equiv 0(\bmod n)$, see Figure 6 . Hence, the maximum order of up-down colorings of $T(i)$ is

$$
\operatorname{maxord}(T(i))=2 i
$$

Hence, by Theorem [1.2, we can see that at least $|i-j|$ RII-moves are needed to transform $T(i)$ to $T(j)$ by using generalized Reidemeister moves. Indeed, $\mid i-$ $j \mid$ coincides with the minimum number of RII-moves needed for transformations between $T(i)$ and $T(j)$.

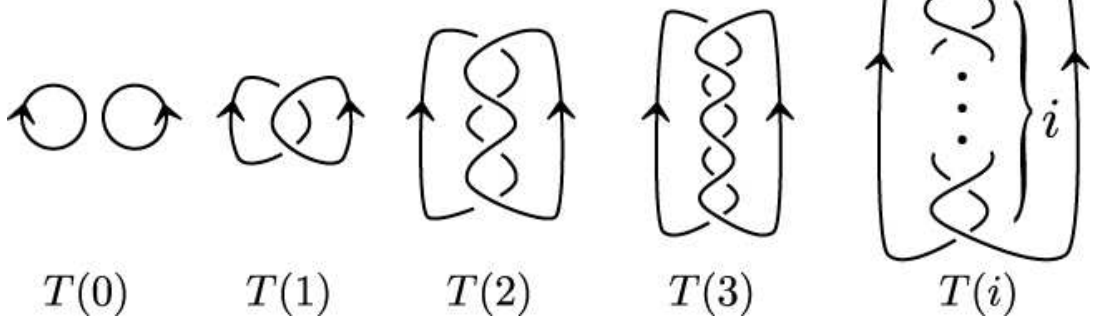

Figure 5. The 2-component virtual-link diagrams $T(i)$.

Example 3.7. For the virtual-link diagrams $T(5)$ and $T^{\prime}(5)$ in Figure 7 , the maximum orders of up-down colorings are

$$
\operatorname{maxord}(T(5))=10 \text { and } \operatorname{maxord}\left(T^{\prime}(5)\right)=6 .
$$

Therefore at least $2(=|10-6| / 2)$ RII-moves are needed to transform $T(5)$ to $T^{\prime}(5)$ by using generalized Reidemeister moves. Indeed, 2 coincides with the minimum number of RII-moves needed for transformations between $T(5)$ and $T^{\prime}(5)$.

Similarly, we can see that the minimum number of RII-moves needed for transformations between $T(5)$ and $T^{\prime \prime}(5)$ (resp. $T^{\prime}(5)$ and $\left.T^{\prime \prime}(5)\right)$ is 4 (resp. 2). 


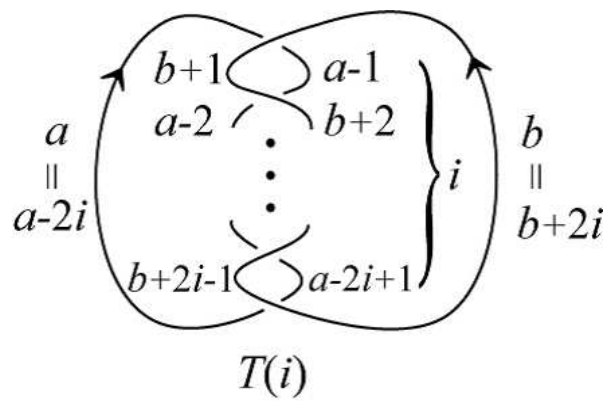

Figure 6 . Colorability of $T(i)$ by up-down colorings..

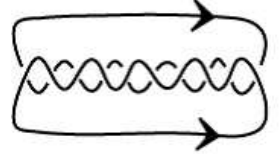

$T(5)$

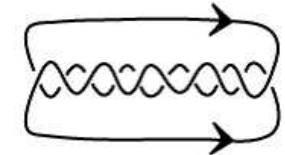

$T^{\prime}(5)$

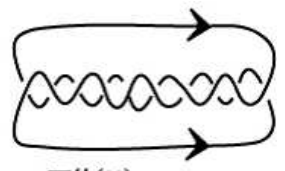

$T^{\prime \prime}(5)$

FiguRE 7. The 2-component virtual-link diagrams $T(5), T^{\prime}(5)$ and $T^{\prime \prime}(5)$.

Remark. 3.8. The minimum number of required RII-moves between given two 2component virtual-link diagrams can be also estimated by using the number of nonself real-crossings since RII-moves only change the number of such real-crossings. More precisely, the half of the difference of the numbers of non-self real-crossings gives a lower bound of the number of required RII-moves. Hence, it is easily seen that two different diagrams $T(i)$ and $T(j)$ in the family $\{T(i)\}_{i \in\{0,1,2, \ldots\}}$ of Example 5 need at least $|i-j|$ RII-moves to be transformed each other. However the minimum number of RII-moves needed for transformations between $T(5)$ and $T^{\prime}(5)$ (or $T^{\prime}(5)$ and $T^{\prime \prime}(5), T(5)$ and $\left.T^{\prime \prime}(5)\right)$ in Figure 7 can not be detected by using the number of non-self real-crossings, but by using our method.

\section{COCYCle INVARIANTS USING UP-DOWN COLORINGS AND THE NECESSITY OF REIDEMEISTER MOVES OF TYPE II}

We note again that we may read this section except for the proof of Theorem 1.4 by replacing "virtual" with "classical" and "generalized Reidemeister moves" with "Reidemeister moves".

In this section, we define a multi-set $\Phi_{f}(D)$ which is analogous to quandle cocycle invariants.

Let $n$ be a positive integer. Let $A$ be an abelian group.

Definition 4.1. An $n$-up-down cocycle is a map $f: \mathbb{Z}_{n} \times \mathbb{Z}_{n} \times\{+,-\} \rightarrow A$ satisfying the following conditions:

- For any $a \in \mathbb{Z}_{n}$ and $\varepsilon \in\{+,-\}$,

(0) $f(a, a, \varepsilon)=0$.

- For any $a, b, c \in \mathbb{Z}_{n}$,

(1) $f(a-1, b,-)+f(b+1, c+1,+)+f(a-1, c+2,+)=f(a-2, b-$ $1,-)+f(b, c+2,+)+f(a, c+1,+)$, 
(2) $f(a-1, b,-)+f(b, c+1,-)+f(a-2, c,-)=f(a-2, b-1,-)+f(b-$ $1, c,-)+f(a-1, c+1,-)$

(3) $f(a-1, b+1,+)+f(b+1, c+1,+)+f(a-1, c+1,-)=f(a, b,+)+$ $f(b, c+2,+)+f(a-2, c,-)$

(4) $f(a-1, b+1,+)+f(b, c+1,-)+f(a, c+1,+)=f(a, b,+)+f(b-$ $1, c,-)+f(a-1, c+2,+)$

(5) $f(a, b+1,+)+f(b+1, c+2,+)+f(a-1, c+1,+)=f(a-1, b,+)+$ $f(b, c+1,+)+f(a, c+2,+)$

(6) $f(a, b+1,+)+f(b, c,-)+f(a-2, c+1,-)=f(a-1, b,+)+f(b-$ $1, c+1,-)+f(a-1, c,-)$,

(7) $f(a-2, b,-)+f(b+1, c+2,+)+f(a-1, c,-)=f(a-1, b-1,-)+$ $f(b, c+1,+)+f(a-2, c+1,-)$ and

(8) $f(a-2, b,-)+f(b, c,-)+f(a, c+2,+)=f(a-1, b-1,-)+f(b-$ $1, c+1,-)+f(a-1, c+1,+)$.

When we do not specify $n$, we call such a map an up-down cocycle for simplicity.

We will later show that the above conditions are related to the RI-moves and the RIII-moves, see the proof of Theorem [1.3. On the other hand, quandle cocycle conditions are also related to the RI-moves and an RIII-move, see [2]. In that sense, we call a map satisfying the above conditions an up-down cocycle. However we do not know if there exists a (co)homology theory which are related to up-down cocycles.

Definition 4.2. An $n$-up-down cocycle $f$ is shiftable if $f(a+1, b+1, \varepsilon)=f(a, b, \varepsilon)$ for any $a, b \in \mathbb{Z}_{n}$ and $\varepsilon \in\{+,-\}$.

Remark. 4.3. A map $f: \mathbb{Z}_{n} \times \mathbb{Z}_{n} \times\{+,-\} \rightarrow A$ is a shiftable $n$-up-down cocycle if and only if it satisfies the following conditions (A)-(C):

(A) For any $a \in \mathbb{Z}_{n}$ and $\varepsilon \in\{+,-\}, f(a, a, \varepsilon)=0$.

(B) For any $a, b \in \mathbb{Z}_{n}$ and $\varepsilon \in\{+,-\}, f(a+1, b+1, \varepsilon)=f(a, b, \varepsilon)$.

(C) For any $a, b, c \in \mathbb{Z}_{n}$ and $\varepsilon \in\{+,-\}$, the following equations (i)-(iii) hold:

(i) $f(b+1, c+1, \varepsilon)+f(a-1, c+2, \varepsilon)=f(b, c+2, \varepsilon)+f(a, c+1, \varepsilon)$,

(ii) $f(a-1, b+1, \varepsilon)+f(b+1, c+1, \varepsilon)=f(a, b, \varepsilon)+f(b, c+2, \varepsilon)$ and

(iii) $f(a-1, b+1, \varepsilon)+f(a, c+1, \varepsilon)=f(a, b, \varepsilon)+f(a-1, c+2, \varepsilon)$.

Example 4.4. Define $f: \mathbb{Z}_{4} \times \mathbb{Z}_{4} \times\{+,-\} \rightarrow \mathbb{Z}_{4}$ by

$$
\begin{gathered}
f(a, b,+)= \begin{cases}0 & (a=b), \\
2 & (a=b+1), \\
2 & (a=b+2), \\
0 & (a=b+3) \text { and }\end{cases} \\
f(a, b,-)= \begin{cases}0 & (a=b), \\
1 & (a=b+1), \\
2 & (a=b+2), \\
3 & (a=b+3) .\end{cases}
\end{gathered}
$$

Then $f$ is a shiftable 4-up-down cocycle.

Let $D$ be a diagram of a virtual-knot and $C$ an $n$-up-down coloring of $D$. Let $f: \mathbb{Z}_{n} \times \mathbb{Z}_{n} \times\{+,-\} \rightarrow A$ be an $n$-up-down cocycle. For each real-crossing $c$ of $D$, 
we define the weight $w_{c}$ of $c$ as follows: Let $u_{1}, u_{2}$ (resp. $o_{1}, o_{2}$ ) be the under-semiarcs (resp. over-semi-arcs) around $c$ such that the orientation of $D$ points from $u_{1}$ to $u_{2}$ (resp. from $o_{1}$ to $o_{2}$ ).

- When $c$ is positive, set $w_{c}=f\left(C\left(u_{1}\right), C\left(o_{2}\right),+\right)$. See Figure 8

- When $c$ is negative, set $w_{c}=f\left(C\left(u_{2}\right), C\left(o_{1}\right),-\right)$. See Figure 8 .
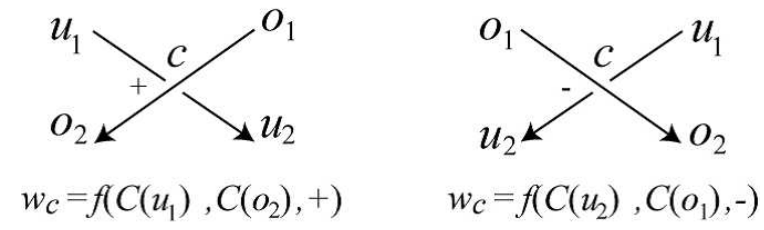

FiguRE 8. The weight of a real-crossing $c$ with respect to an updown coloring.

Let $W_{f}(D, C)$ be the sum of the weights for all the real-crossings of $D$, that is,

$$
W_{f}(D, C)=\sum_{c} w_{c}
$$

We denote by $\Phi_{f}(D)$ the multi-set

$$
\left\{W_{f}(D, C) \mid C \in\{n \text {-up-down colorings of } D\}\right\} \text {. }
$$

Theorem 1.3 implies that the multi-set $\Phi_{f}(D)$ is useful to detect the necessity of RII-moves.

Proof of Theorem 1.3. Let $D$ and $D^{\prime}$ be virtual-knot diagrams such that $D^{\prime}$ is obtained from $D$ by a single RI-move. For an $n$-up-down coloring $C$ of $D$, we set an $n$-up-down coloring $C^{\prime}$ of $D^{\prime}$ so that $C$ is corresponding to $C^{\prime}$ by the bijection defined in the proof of Theorem 1.1. Then as shown in Figure 13, we have

$$
W_{f}(D, C)-W_{f}\left(D^{\prime}, C^{\prime}\right)= \pm f(a, a, \varepsilon)
$$

for some $a \in \mathbb{Z}_{n}$ and $\varepsilon \in\{+,-\}$. By the condition (0) of an $n$-up-down cocycle, since $f(a, a, \varepsilon)=0, W_{f}(D, C)=W_{f}\left(D^{\prime}, C^{\prime}\right)$ holds.

Next let us consider the cases of RIII-moves. As shown in Figure 14, we have eight RIII-moves (1)-(8). Let $D$ and $D^{\prime}$ be virtual-knot diagrams such that $D^{\prime}$ is obtained from $D$ by the RIII-move (1) in Figure 14, For an $n$-up-down coloring $C$ of $D$, we set an $n$-up-down coloring $C^{\prime}$ of $D^{\prime}$ so that $C$ is corresponding to $C^{\prime}$ by the bijection defined in the proof of Theorem 1.1. Then we have

$$
\begin{aligned}
W_{f}(D, C)- & W_{f}\left(D^{\prime}, C^{\prime}\right) \\
= \pm\{ & (f(a-1, b,-)+f(b+1, c+1,+)+f(a-1, c+2,+)) \\
& -(f(a-2, b-1,-)+f(b, c+2,+)+f(a, c+1,+))\}
\end{aligned}
$$

for some $a, b, c \in \mathbb{Z}_{n}$, see Figure 14, By the condition (1) of an $n$-up-down cocycle, since the right side of the above equation is equal to 0 , we have $W_{f}(D, C)=$ $W_{f}\left(D^{\prime}, C^{\prime}\right)$. Similarly, in the cases of the other RIII-moves (2)-(8), by the conditions (2)-(8) of an $n$-up-down cocycle, respectively, we have $W_{f}(D, C)=W_{f}\left(D^{\prime}, C^{\prime}\right)$.

It is obvious that the other generalized Reidemeister moves other than the RI I-moves also do not change the weight sum $W_{f}(D, C)$, see Figure 15 for the VRI V-moves. 
As a consequence, we have a bijection $\Phi_{f}(D) \rightarrow \Phi_{f}\left(D^{\prime}\right), W_{f}(D, C) \mapsto W_{f}\left(D^{\prime}, C^{\prime}\right)$ such that $W_{f}(D, C)=W_{f}\left(D^{\prime}, C^{\prime}\right)$ for each of the generalized Reidemeister moves other than the RII-moves.

Now we assume that the $n$-up-down cocycle $f$ is shiftable. Then the value $W_{f}(D, C)$ does not depend on the choice of the $n$-up-down coloring $C$, see Figure 9 and Corollary 3.5. Hence, we denote by $\Phi_{f}^{\text {shift }}(D)$ the value $W_{f}(D, C)$ for some
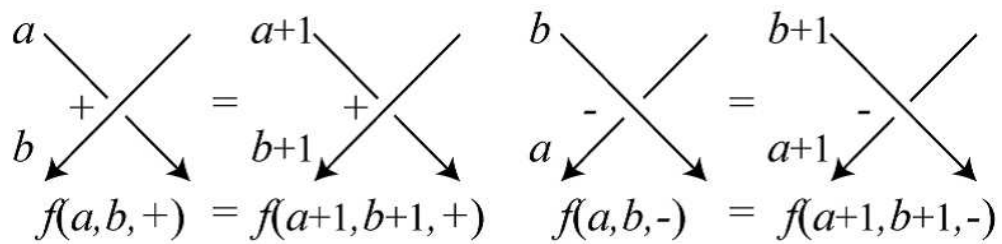

FIGURE 9. The weight of a real-crossing with respect to up-down colorings $C$ and $C+1$

$n$-up-down coloring $C$. Note that $\Phi_{f}(D)$ and $\Phi_{f}^{\text {shift }}(D)$ are essentially the same. Next property is obtained as a corollary of Theorem 1.3 .

Corollary 4.5. Let $D$ and $D^{\prime}$ be diagrams which represent the same virtual-knot. If $\Phi_{f}^{\text {shift }}(D) \neq \Phi_{f}^{\text {shift }}\left(D^{\prime}\right)$, then any sequence of generalized Reidemeister moves between $D$ and $D^{\prime}$ includes at least one RII-move.

Lemma 4.6. Let $D_{1}$ and $D_{2}$ be virtual-knot diagrams. For any shiftable up-down cocycle $f$ and for any connected sum $D=D_{1} \sharp D_{2}$ of $D_{1}$ and $D_{2}$, the equality

$$
\Phi_{f}^{\text {shift }}(D)=\Phi_{f}^{\text {shift }}\left(D_{1}\right)+\Phi_{f}^{\text {shift }}\left(D_{2}\right)
$$

holds.

Proof. Let $n$ be an positive integer. Let $C$ be an $n$-up-down coloring of $D$. Then $C$ is separated into an $n$-up-down coloring $C_{1}$ of $D_{1}$ and an $n$-up-down coloring $C_{2}$ of $D_{2}$ such that $C_{i}(i=1,2)$ satisfies that $C_{i}(e)=C\left(e^{\prime}\right)$ if $e \cap e^{\prime} \neq \emptyset$ for $e \in \mathcal{S} \mathcal{A}\left(D_{i}\right)$ and $e^{\prime} \in \mathcal{S} \mathcal{A}(D)$. Hence by the definition of the sum of weights, we have

$$
\Phi_{f}^{\text {shift }}(D)=W_{f}(D, C)=W_{f}\left(D_{1}, C_{1}\right)+W_{f}\left(D_{2}, C_{2}\right)=\Phi_{f}^{\text {shift }}\left(D_{1}\right)+\Phi_{f}^{\text {shift }}\left(D_{2}\right) .
$$

Example 4.7. Let $\Delta$ and $O$ denote the virtual-knot diagrams shown in Figure 10. Note that both of them represent the trivial virtual-knot and $O$ has some orientation. Let $f: \mathbb{Z}_{4} \times \mathbb{Z}_{4} \times\{+,-\} \rightarrow \mathbb{Z}_{4}$ be the shiftable 4 -up-down cocycle in
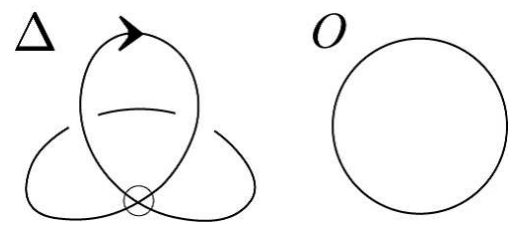

Figure 10. Virtual knot diagrams $\Delta$ and $O$. 
Example 4.4. We set the 4-up-down coloring as shown in Figure 10. Then the sum of the weights of the real-crossings of $\Delta$ is

$$
f(1,0,-)+f(1,2,+)=1+0=1 .
$$

Hence we have $\Phi_{f}^{\text {shift }}(\Delta)=1$. On the other hand, since $O$ has no real-crossing, $\Phi_{f}^{\text {shift }}(O)=0$ holds. Therefore by Corollary 4.5, at least one RII-move is needed to transform $\Delta$ to $O$.

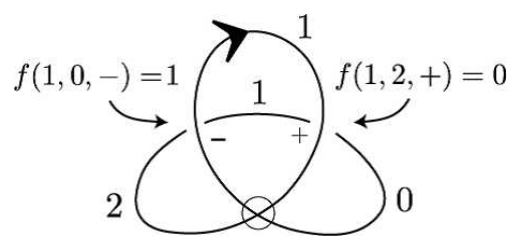

Figure 11. $\Phi_{f}^{\text {shift }}(\Delta)=1$

Proof of Theorem 1.4Let $D^{\prime}$ be a virtual-knot diagram which is obtained by taking the connected sum $\Delta \sharp D$ depicted in Figure 12, where we may replace the diagram $\Delta$ with the one obtained after performing a single RI-move as shown in the lower picture of Figure 12. Then $D$ and $D^{\prime}$ represent the same virtual-knot.

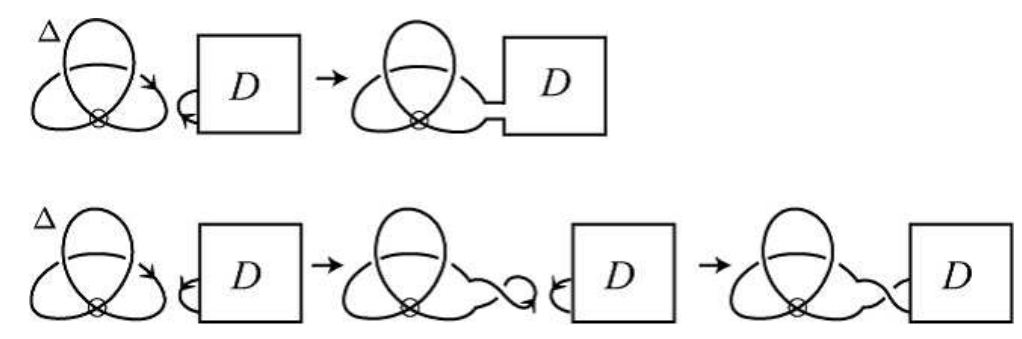

Figure 12. A connected sum.

By Lemma 4.6 and Example 4.7, we have

$$
\Phi_{f}^{\text {shift }}\left(D^{\prime}\right)=\Phi_{f}^{\text {shift }}(\Delta)+\Phi_{f}^{\text {shift }}(D)=1+\Phi_{f}^{\text {shift }}(D) \neq \Phi_{f}^{\text {shift }}(D) \text { in } \mathbb{Z}_{4} .
$$

Therefore by Corollary 4.5, at least one RII-move is needed to transform $D$ to $D^{\prime}$.

Remark. 4.8. The unoriented version of Theorem 1.4 also holds. To prove this, we choose a shiftable $n$-up-down cocycle $g$ instead of the 4-up-down cocycle $f$ in Example 4.4 such that for the virtual-knot diagram $\Delta$ in Figure 10, $\Phi_{g}^{\text {shift }}(\Delta) \neq 0$ and for any virtual-knot diagram $D, \Phi_{g}^{\text {shift }}(D)$ does not depend on the orientation of $D$. Then we can see that for the virtual-knot diagrams $D$ and $D^{\prime}=\Delta \sharp D$ with any orientations, $\Phi_{g}^{\text {shift }}(D) \neq \Phi_{g}^{\text {shift }}\left(D^{\prime}\right)$, which implies that as the unoriented virtual-knot diagrams $D$ and $D^{\prime}$, at least one RII-move is needed for transformations 
between them. We leave the detailed proof to the reader. For example, use the 4-up-down cocycle $g: \mathbb{Z}_{4} \times \mathbb{Z}_{4} \times\{+,-\} \rightarrow \mathbb{Z}_{4}$ defined by

$$
g(a, b, \varepsilon)= \begin{cases}1 & (a=b \pm 1, \varepsilon=-) \\ 0 & \text { (otherwise) }\end{cases}
$$

\section{Questions And Future STUdies}

1. (To study about a generalization of up-down colorings.)

Let $n$ be a nonnegative integer and $P$ and $N$ integers.

An $(n ; P, N)$-up-down coloring of a virtual-link diagram $D$ is a map $C: \mathcal{S A}(D) \rightarrow$ $\mathbb{Z}_{n}$ satisfying the following conditions: For a real-crossing $c$ of $D$, let $u_{1}, u_{2}$ (resp. $o_{1}, o_{2}$ ) be the under-semi-arcs (resp. over-semi-arcs) around $c$ such that the orientation of $D$ points from $u_{1}$ to $u_{2}$ (resp. from $o_{1}$ to $o_{2}$ ). Then

- if $c$ is positive,

$$
C\left(u_{2}\right)=C\left(u_{1}\right)-P \text { and } C\left(o_{2}\right)=C\left(o_{1}\right)+P,
$$

and

- if $c$ is negative,

$$
C\left(u_{2}\right)=C\left(u_{1}\right)-N \text { and } C\left(o_{2}\right)=C\left(o_{1}\right)+N .
$$

It is easily seen that $(n ; P, N)$-up-down colorings are a generalization of up-down colorings. Moreover the numbers of $(n ; P, N)$-up-down colorings of virtual-link diagrams can be used to detect the necessity of RII-moves. In addition, the argument in Section 4 is also extended in the cases using $(n ; P, N)$-up-down colorings. We aim to address these topics including some applications in future work. (Moreover note that an $(n ; P, N)$-up-down coloring is a biquandle coloring if and only if $P=-N$.)

2. (To find a (co)homology theory related to up-down cocycles.)

In [2], a quandle cohomology theory was introduced, see also 4, 5]. The 2- or 3 -cocycles (or 3- or 4-cocycles) are used to define a quandle cocycle invariant of an oriented classical-link (or an oriented surface-link). The cocycle conditions are related to the RI-moves and an RIII-move, see [2].

In Section 4 we defined an up-down cocycle. The cocycle conditions are also related to the RI-moves and all the RIII-moves. However we do not know if there exists a (co)homology theory which are related to up-down cocycles. Hence, it might be natural to ask the following question:

Question 5.1. Is there a cohomology theory whose 2-cycles are related to the updown cocycles?

3. (To find an application for classical-knots.)

In Section 4, by our method, we found two trivial virtual-knot diagrams such that at least one RII-move is needed for transformations between them. For classical-knot diagrams, we give the following question:

Question 5.2. Is there a pair of trivial classical-knot diagrams such that at least one RII-move are needed for transformations between them and the necessity of RI I-moves is detected by our method (, but not detected by Hagge's or Manturov's one)?

If we find such a pair, we might be able to give a new example satisfying the following theorem: 


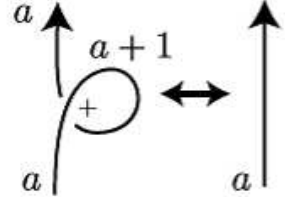

$f(a+1, a+1,+)=0$

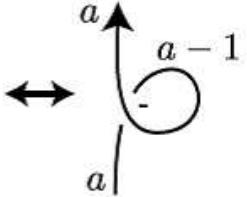

$f(a-1, a-1,-)=0$

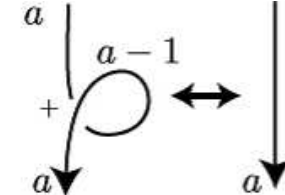

$f(a, a,+)=0$

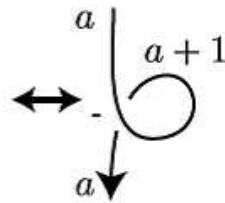

$f(a, a,-)=0$

Figure 13. The RI-moves.

Theorem 5.3. (cf. 6]) For any classical-knot diagram D, there exists a diagram $D^{\prime}$ representing the same classical-knot such that any sequence of Reidemeister moves between $D$ and $D^{\prime}$ includes at least one RII-move.

4. (To study about the other generalized Reidemeister moves.)

Question 5.4. For each $\mathcal{M} \in\{R I, R I I, R I I I, V R I, V R I I, V R I I I, V R I V\}$, is there a pair of trivial virtual-knot diagrams such that at least one $\mathcal{M}$-move is needed for transformations between them?

When we restrict the moves $\mathcal{M}$ to $\mathcal{M} \in\{R I, R I I, R I I I, V R I\}$, the statement of the above question is true, see $[3,6,12,13,16$. Note that it is easy to construct a pair of trivial virtual-knot diagrams which need at least one VRI-move between them, since the parity of the number of virtual-crossings is unchanged under the generalized Reidemeister moves except for the VRI-move. If we find such a pair of trivial virtual-knot diagrams for the other generalized Reidemeister moves, we might be able to answer the next conjecture:

Conjecture. 5.5. For any virtual-knot $K$, there exist two diagrams $D$ and $D^{\prime}$ of $K$ such that any sequence of generalized Reidemeister moves between $D$ and $D^{\prime}$ must contain all of the generalized Reidemeister moves.

\section{REFERENCES}

[1] J. S. Carter, M. Elhamdadi, M. Saito and S. Satoh: A lower bound for the number of Reidemeister moves of type III, Topology and its Applications 153 (2006), 2788-2794.

[2] J. S. Carter, D. Jelsovsky, S. Kamada, L. Langford and M. Saito: Quandle cohomology and state-sum invariants of knotted curves and surfaces, Trans. Amer. Math. Soc. 355 (2003), no. 10, 3947-3989.

[3] Z. Cheng and H. Gao: A note on the independence of Reidemeister moves, J. Knot Theory Ramifications 21 (2012), no. 9, 1220001, 7 pp.

[4] R. Fenn, C. Rourke and B. Sanderson: Trunks and classifying spaces, Appl. Categ. Structures 3 (1995), no. 4, 321-356.

[5] R. Fenn, C. Rourke and B. Sanderson: James bundles and applications, preprint (1996), http://www.maths.warwick.ac.uk/ $\mathrm{cpr} / \mathrm{ftp} /$ james.ps.

[6] T. J. Hagge: Every Reidemeister move is needed for each knot type, Proc. Amer. Math. Soc. 134 (2005), 295-301.

[7] J. Hass and T. Nowik: Invariants of knot diagrams, Math. Ann. 342 (2008), 125-137.

[8] C. Hayashi: A lower bound for the number of Reidemeister moves for unknotting, J. Knot Theory Ramifications 15 (2006), 313-325.

[9] C. Hayashi, M. Hayashi and T.Nowik: Unknotting number and number of Reidemeister moves needed for unknotting, Topology and its Applications 159 (2012), 1467-1474.

[10] L. H. Kauffman: Virtual knot theory, European J. Combin. 20 (1999), 663-690.

[11] A. M. Lowrance: Alternating distance of knots and links, Topology and its applications 182 (2015), 53-70. 

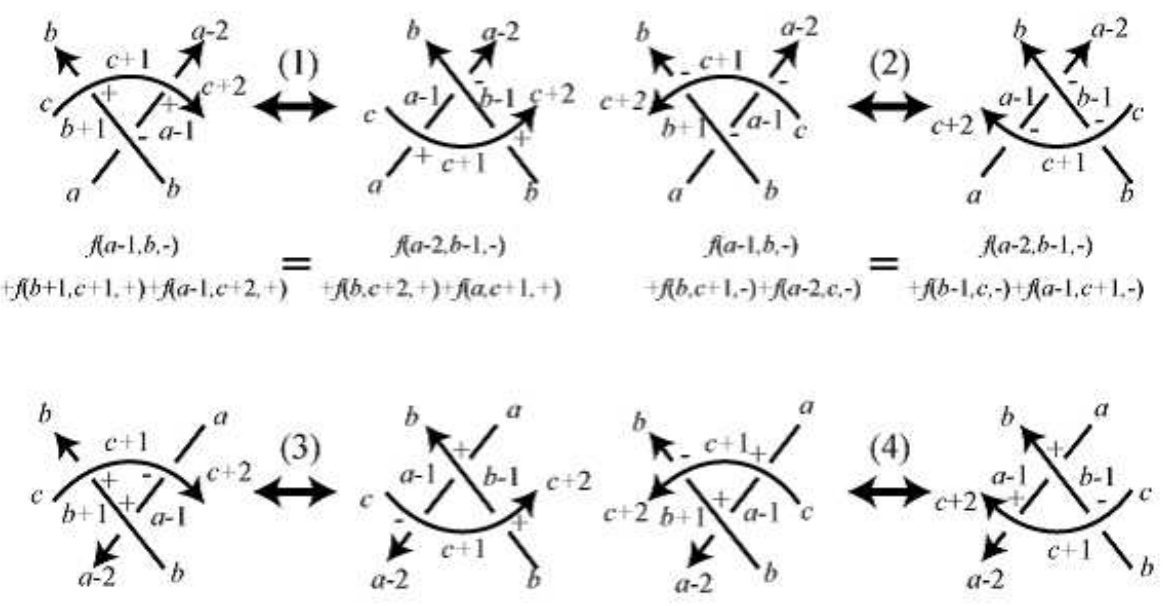

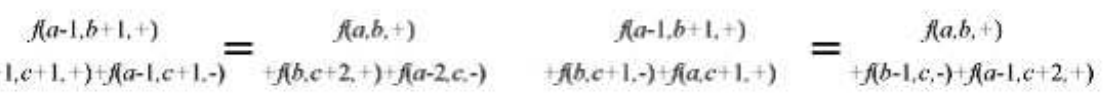
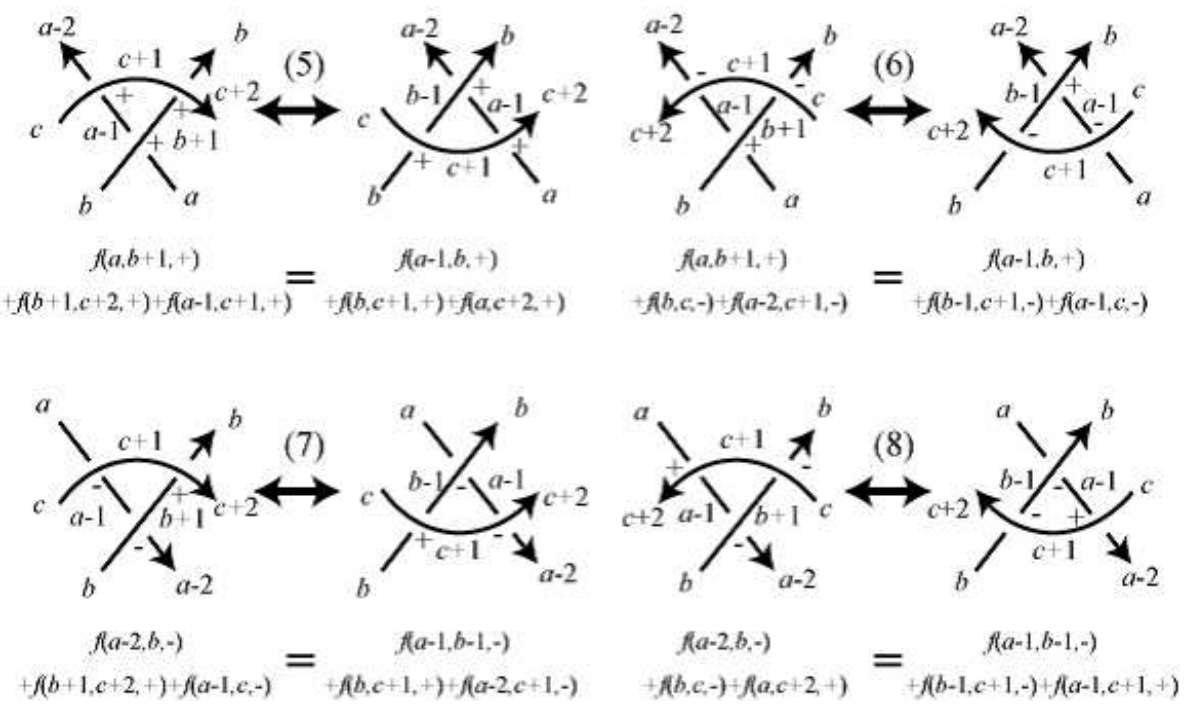

Figure 14. The RIII-moves.

[12] V. O. Manturov: Knot Theory, Chapman \& Hall/CRC, Boca Raton, FL (2004), xiv+400 pp.

[13] O. P. Östlund: Invariants of knot diagrams and relations among Reidemeister moves, J. Knot Theory Ramifications 10 (2001), 1215-1227.

[14] K. Reidemeister: Elementare Begründung der Knotentheorie, Abh. Math. Sem. Univ. Hamburg. 5 (1927) 24-32.

[15] A. Shimizu: The warping polynomial of a knot diagram, J. Knot Theory Ramifications 21 (2012), 1250124, $15 \mathrm{pp}$.

[16] B. Trace: On the Reidemeister moves of a classical knot, Proc. Amer. Math. Soc. 89 (1983), $722-724$. 


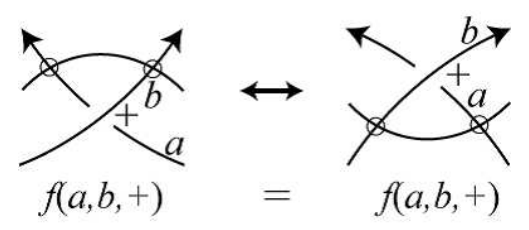

FigURE 15. VRIV-moves

Deparment of Information and Communication Sciences, Sophia University, 7-1 KioiCHO, CHIYODA-KU, TOKYO 102-8554, JAPAN

E-mail address: oshirok@sophia.ac.jp

Department of Mathematics, National Institute of Technology, Gunma College, 580 TORIBA-Cho, MaEbashi-Shi, Gunma 371-8530, JaPAN

E-mail address: shimizu@nat.gunma-ct.ac.jp

Department of Mathematics, National Institute of Technology, Gunma College, 580 TORIBA-Cho, MaEbashi-Shi, Gunma 371-8530, Japan

E-mail address: yaguchi-y@nat.gunma-ct.ac.jp 